\title{
Light-scattering-based analysis of biomolecular interactions
}

\author{
Daniel Some
}

Received: 26 December 2012 / Accepted: 31 January 2013 /Published online: 6 March 2013

(C) The Author(s) 2013. This article is published with open access at Springerlink.com

\begin{abstract}
While light scattering has long been applied to the analysis of biomolecular interactions, recent advances have extended the practical use of light scattering techniques to cover a rather broad range of phenomena. In this paper I review essential light scattering theory as applied to specific interactions under thermodynamically ideal conditions and present examples showing how light scattering elucidates the dynamic equilibrium and kinetic behavior of proteins and other biomacromolecules.
\end{abstract}

Keywords Light scattering · Interactions · CG-MALS · CG-DLS · Affinity · Stoichiometry

\section{Introduction}

Light scattering (LS) is a versatile tool for characterizing biomacromolecules and their interactions, affording solution-based measurements of dynamic equilibrium properties and kinetic phenomena without recourse to immobilization or labeling. While both flavors of light scatteringmulti-angle static light scattering (SLS or MALS) and dynamic light scattering (DLS) - are of great utility, the power of MALS, in particular for these types of studies, lies in the combination of practical and conceptual simplicity, theoretical and analytical rigor, and generality.

In vitro interaction measurements comprise two essential elements: a series of solutions containing the interacting species at different concentrations or compositions, and a reporter signal which is modulated by the degree of interaction. While most techniques involve reporter signals that are only

Special Issue: Protein-Protein and Protein-Ligand Interactions in Dilute and Crowded Solution Conditions. In Honor of Allen Minton's 70th Birthday.

D. Some $(\bowtie)$

Wyatt Technology Corp, 6300 Hollister Ave,

Santa Barbara, CA 93117, USA

e-mail: dsome@wyatt.com indirectly related to the interaction product (e.g., fluorescence intensity, 'response units', consumed enzyme, etc.), MALS reports the weight-average solution molar mass $\mathrm{M}_{\mathrm{w}}$, which is directly connected with the essence of complex formation. To illustrate, consider the 1:1 association of an equimolar solution of two proteins, each with molar mass $50 \mathrm{kDa}$. In the absence of interaction, $\mathrm{M}_{\mathrm{w}}=50 \mathrm{kDa}$, while under stoichiometric association $\mathrm{M}_{\mathrm{w}}=100 \mathrm{kDa}$-leading to a doubling of the MALS signal.

With minimal investment in sample preparation, method development, and data analysis, light scattering yields rich information, including molar mass, binding affinity, and absolute stoichiometry of complex interactions, such as simultaneous homo- and hetero-association or specific multi-valent complex formation, the magnitude of nonspecific interactions, the size (radius of gyration $r_{g}$ or hydrodynamic radius $r_{h}$ ) of these complexes, and the quantitative kinetics of binding, aggregation, dissociation or degradation. The emergence of new instrumentation for automating and facilitating these types of studies holds promise for re-invigorating and expanding the use of both MALS and DLS in biomolecular interaction research, complementing other label-free biophysical techniques.

Qualitative or semi-quantitative turbidity and $90^{\circ}$ light scattering are commonly used to monitor aggregation kinetics and equilibrium. The focus of this review will be quantitative analyses of light scattering to determine binding affinity, stoichiometry and/or detailed kinetics, and in particular recent developments resulting from automated and highly sensitive MALS and DLS instruments.

\section{Theoretical background}

Biomolecules are subject to various types of interactions. One important classification divides specific interactionsthose that lead to the formation of well-defined complexes via site-specific binding-from non-specific interactions arising from net charge, dipoles, hydrophobic patches, van 
der Waals forces, etc. The thermodynamic (and, consequentially, light-scattering) treatment of specific interactions differs from that of non-specific interactions. Specific interactions are considered to produce discrete species, coexisting in dynamic equilibrium with free monomers and modeled as a composition-dependent polydisperse solution. Non-specific interactions are modeled as thermodynamic non-ideality; these are not mutually exclusive and may occur in tandem. In this section I focus on MALS; the interested reader is referred to Hanlon et al. (2010) for the composition-gradient (CG)-DLS theory of specific binding.

Specific reversible association in the ideal limit

Some examples of modeling static light scattering measurements for specific reversible associations were discussed by Burchard and Cowie (1972). These were generalized by Attri and Minton $(2005 \mathrm{a}, \mathrm{b})$ and are reproduced with minor notational changes below.

Details of the assumptions incorporated in MALS analysis appear in Wyatt (1993) and Attri and Minton (2005a). To summarize, the 'ideal gas law' for static light scattering from a solution comprising multiple, non-interacting species $i$ (i.e., species not subject to appreciable non-specific interactions/thermodynamic non-ideality), presented in Eq. 1, is simply a sum of terms, each of which corresponds to the scattered intensity from an individual species.

$$
\frac{R(\theta)}{K}=\sum_{i} \frac{R_{i}(\theta)}{K}=\sum_{i} M_{i} c_{i} P\left(\theta, r_{g, i}\right)\left(\frac{d n}{d c_{i}}\right)^{2}
$$

$R(\theta)$ represents the excess Rayleigh ratio (a quantity related to the time-averaged intensity of light scattered by the solute) measured at each detector angle $\theta$, and $R_{i}$ is the excess Rayleigh ratio due to each species in solution. $K$ is a system-dependent constant. $M_{i}, c_{i}$, and $d n / d c_{i}$ refer to the molar mass, mass/volume concentration, and refractive increment of each species $i$, respectively. $P\left(\theta, r_{g, i}\right)$ is the dependence of the scattered light on azimuth angle $\theta$, as given by Eq. 2 .

$P\left(\theta, r_{g}\right)=1-\frac{16 \pi^{2} n_{0}^{2}}{3 \lambda_{0}^{2}}\left\langle r_{g}^{2}\right\rangle \sin ^{2}\left(\frac{\theta}{2}\right)+O\left(\sin ^{4}\left(\frac{\theta}{2}\right)\right)$

The quantity $r_{g}=\sqrt{\left\langle r_{g}^{2}\right\rangle}$ is the molecular mean square radius defined as $\sqrt{\int r^{2} d m / \int d m}$. For most proteins and other molecules smaller than a few hundreds of kiloDaltons, $r_{g}<<\lambda_{0} / n_{0} ; P \approx 1$ and angular dependence may be ignored. Otherwise, measurements of $P(\theta)$ are extrapolated to $\theta=0$ to relate $R(\theta)$ to molar masses.
Specific binding of up to two distinct biomacromolecules $X$ and $Y$ is characterized in equilibrium by ideal light scattering (Eq. 3, transformed from Eq. 1) and mass action (Eq. 4). The solution is considered to consist of a mixture of discrete macromolecular species: free monomers $\mathrm{X}$ $\left(X_{1} Y_{0}\right)$ and $\mathrm{Y}\left(X_{0} Y_{1}\right)$, and one or more complexes $X_{i} Y_{j}$. The set of equations describing the association model is completed by the conservation-of-mass Eq. 5 .

$$
\begin{aligned}
& \frac{R(0)}{K}=\sum_{i, j}\left(i M_{X} \frac{d n}{d c_{X}}+j M_{Y} \frac{d n}{d c_{Y}}\right)^{2}\left[X_{i} Y_{j}\right] \\
& i X+j Y \stackrel{K_{A}^{i j}}{\rightleftarrows} X_{i} Y_{j} ; \quad K_{A}^{i j}=\frac{\left[X_{i} Y_{j}\right]}{[X]^{i}[Y]^{j}} \\
& {[X]^{\text {tot }}=\sum_{i} i\left[X_{i} Y_{j}\right] ; \quad[Y]^{t o t}=\sum_{j} j\left[X_{i} Y_{j}\right]}
\end{aligned}
$$

Non-specific interactions/non-ideality

Non-specific interactions in biomolecular systems are usually weak in comparison to specific interactions. Typical proteins and other compact biomacromolecules exhibit negligible non-ideality below concentrations of $\sim 1 \mathrm{~g} / \mathrm{L}$, although a lower concentration limit for ideal behavior applies to charged molecules in poorly screened buffers as well as those presenting diffuse or extended structures. Optimal CG-MALS measurements cover a concentration range of $\sim 0.1-10 \times \mathrm{K}_{\mathrm{D}}$, corresponding to an upper $\mathrm{K}_{\mathrm{D}}$ of $10 \mu \mathrm{M}$ (for $10-\mathrm{kDa}$ molecules) or $1 \mu \mathrm{M}$ (for $100-\mathrm{kDa}$ molecules) that may be characterized entirely in the ideal limit; the majority of examples in this review fall within this range. Any analysis of specific binding above the ideal concentration limit must account for non-ideality; likewise, non-specific interactions must generally be analyzed at minimum concentrations of a few grams per liter.

While CG-MALS is well-suited to the analysis of nonspecific interactions and also to the analysis of specific or quasi-specific interactions in the presence of thermodynamic non-ideality, the theoretical description is beyond the scope of this review. For further details, the reader is referred to Minton (2007) and Fernández and Minton (2008, 2009).

\section{Quasi-equilibrium techniques}

Light scattering measurements are conducted in both fractionated mode and unfractionated or batch mode. Fractionation, usually accomplished by means of size exclusion chromatography (SEC) or asymmetric field-flow 
fractionation, has the advantage of separating out from the sample of interest any large aggregates and other particulates that are highly detrimental to light scattering signals. Wyatt (1993) reviewed the utility of SEC coupled to MALS for absolute characterization of molar mass and size distributions, independent of column calibration. This technique can assess the stoichiometry of tightly bound complexes that do not dissociate upon chromatographic dilution or column shear. For interaction assays the disadvantage of fractionation lies in the ongoing dilution and separation of molecules that must occupy the same volume in order to interact. This leads to illdefined states in terms of kinetics or dynamic equilibrium, so that fully quantitative interaction analysis in the fractionated mode is only possible under certain carefully characterized conditions: either very rapid or very slow equilibration (on the time scale of the separation).

Several authors have reported quantifying monomer-dimer interactions by SEC-MALS. Das et al. (2008) studied SecA and SecA mutants, analyzing a series of chromatography peaks injected at successive concentrations, assuming fast equilibration. Equilibrium dissociation constants $\left(\mathrm{K}_{\mathrm{D}}\right)$ of less than $100 \mathrm{nM}$ to over $10 \mu \mathrm{M}$ were characterized. A similar analysis was performed by Kapoor et al. (2010) on nucleobindin-1; these authors also estimated stoichiometry and binding affinity of nucleobindin-1 with G-protein $\alpha$ subunits by injecting a series of mixtures at the appropriate molar ratios. Bajaj et al. (2007) demonstrated an integrated LS-UV cell to overcome the technical problem of inter-detector dilution, determining the $K_{D}$ of $\beta$-lactoglobulin dimerization.

\section{Equilibrium techniques}

Ideally, interaction studies should be performed under true equilibrium, or at least well-defined kinetic states. The series of batch MALS or DLS measurements over varying compositions required to analyze affinity and stoichiometry is referred to as composition-gradient MALS (CG-MALS) or DLS (CG-DLS). Approaches to batch measurements differ in levels of automation and the type of non-equilibrium data that may be acquired on the way to equilibrium. CG-MALS and CGDLS require minimal method development. A typical automated analysis takes $0.5-3 \mathrm{~h}$ (depending on equilibration rates), and usually only one or two such runs are required to reach full characterization.

\section{Apparatus}

\section{Cuvette}

Cuvette-based measurements have been the mainstay of CG-MALS and CG-DLS for decades. Samples are prepared at the requisite concentrations or compositions, placed in scintillation vials or square microcuvettes, then measured individually by placing the cuvette into a MALS or DLS detector. Concentrations must be measured off-line or on the basis of accurate dilutions of a known stock. Scintillation vials afford full multi-angle measurements but require copious amounts of sample and, due to the poor optical quality and mechanical tolerances, introduce relatively large uncertainty. High-quality, non-disposable square microcuvettes may require only a few microliters of sample but are limited to $90^{\circ}$ scattering and also need to be cleaned thoroughly between samples. In a recent example of cuvette-based CGMALS, Scherer et al. (2010) examined the weak selfassociation of monoclonal antibodies at high concentration versus ionic strength. Due to the high viscosity of these solutions, they were prepared individually and measured in scintillation vials.

\section{Microbatch}

A flow cell provides a controlled and stable environment, low stray light, and superior optical quality for multi-angle measurements, making it preferable to most cuvette-based measurements. In a microbatch experiment, samples are prepared at the requisite compositions and loaded into syringes. A general-use syringe pump dispenses the contents of each syringe through a filter into the MALS/DLS detector. The in-line filter greatly improves signal-to-noise by removing dust particles and large aggregates. An additional advantage of microbatch over cuvette-based measurements is the option for adding an in-line concentration detector instead of relying on off-line measurement or dilution accuracy.

Measurement over a wide range of concentrations is important to capturing the full behavior and should include conditions around the onset of association as well as those producing appreciable oligomer fractions. Woll et al. (2011) analyzed homodimerization of the $\sim 20-\mathrm{kDa}$ neuronal calcium sensor-1 via microbatch measurements with monomerequivalent concentrations that ranged from single-digit micromolar concentrations up to $700 \mu \mathrm{M}$ in order to quantify $\mathrm{K}_{\mathrm{D}}$ of $10 \mu \mathrm{M}$; sedimentation equilibrium (SE) analysis was within the same affinity range at $\mathrm{K}_{\mathrm{D}}=2 \mu \mathrm{M}$.

The tetrameric form of hemoglobin $(\mathrm{Hb})$ is well-known and provides the opportunity to test CG-MALS analysis of a system that exceeds the complexity of simple monomerdimer equilibrium. Yamaguchi and Adachi (2002) employed microbatch measurements to calculate the monomer-dimer and dimer-tetramer $\mathrm{K}_{\mathrm{D}}$ 's of $\mathrm{Hb} \mathrm{A}, \mathrm{Hb} \mathrm{F}$, and also singleglobulin complexes comprising $\alpha$-, $\beta$ - or $\gamma$-globulins. The ability to readily distinguish between the solution behavior of variants suggests that CG-MALS interaction analyses, in combination with a finite number of site-specific mutations, can potentially complement atomic-level structure 
calculations to map out the free-energy landscape across the binding site.

\section{Automated stopped-flow}

Automated stopped-flow CG-MALS/DLS incorporates the advantages of a closed flow-cell-based system with automated sample preparation and delivery. A stopped-flow CGMALS system consists of a multi-angle static light scattering detector, a composition-gradient delivery system, and an optional concentration detector (UV/Vis absorption, differential refractive index, or similar). Since each injection consists of freshly mixed aliquots, association, dissociation, or aggregation kinetics may be observed, although in many systems equilibration occurs too rapidly.

Attri and Minton (2005a, b) described an apparatus utilizing a dual-syringe pump, combining on-the-fly mixing with in-line filtration. The pump was programmed to draw on one or two protein stock solutions or buffer to produce a series of compositions which were delivered to sensitive MALS and UV/Vis absorption detectors. This work quantified sub-micromolar to millimolar $\mathrm{K}_{\mathrm{D}}$ and the stoichiometry of small protein complexes, such as chymotrypsin (CTR) homodimers and the soybean trypsin inhibitor (STI)-CTR hetero-association.

Further evolution of the system, described in Some and Kenrick (2012), has resulted in a commercial triplesyringe-pump system that extends the range of sensitivity to sub-nanomolar affinities, bundled with a complete software package for experiment design, control, data acquisition, and analysis (Calypso, Wyatt Technology, Santa Barbara, CA). Much of the work described below employed either the Attri and Minton configuration or the Calypso system and software in combination with a DAWN-HELEOS MALS detector (Wyatt Technology) and a Waters 2487 UV/Vis absorption detector (Waters Corp, Milford, MA). Practical considerations regarding detector and in-line filter configurations are discussed by Some and Kenrick (2012).

\section{Automated titration}

An automated in-place titration setup was reported by Fernández and Minton (2008). In this apparatus, the stock solution is placed in a cuvette directly inside the MALS detector. A dual syringe pump is programmed to produce a dilution series through successive steps of removing a volume of solution from the cuvette, adding an equal volume of buffer, then mixing by means of a magnetic stir bar. Concentrations are calculated from the known initial concentration and dilution parameters. The same apparatus includes a thermal jacket for temperature control and relatively rapid temperature jumps.

\section{Plate reader}

Dynamic light scattering measures diffusion constants, which may be related to an effective size - the 'hydrodynamic radius' $r_{h}$-via the Stokes-Einstein equation; CGDLS relates changes in solution-average $r_{h}$ to complex formation although it can also be used to evaluate certain conformational changes. Plate reader-based CG-DLS is a quite attractive technique in terms of time, sample quantity, disposable optical cells (microwell plates), and temperature control to rapidly assess thermodynamic parameters such as enthalpy, entropy, and conformational transitions. While Sharma et al. (2008) employed manual batch CG-DLS to evaluate stoichiometry and $K_{D}$ of the fibroblast growth factor-receptor complex, Hanlon et al. (2010) automated multiple CG-DLS experiments - with temperature rampsby means of a DLS plate reader (Wyatt Technology).

The primary disadvantage of CG-DLS is reduced rigor in data analysis: the relationship between the diffusion constants of monomers and their complexes depends greatly on the specific assembly conformation, unlike the strict molar mass relationships analyzed in CG-MALS. CG-DLS covers a smaller range of $\mathrm{K}_{\mathrm{D}}$ compared to CGMALS and, in a plate reader, all but the slowest kinetics are lost. Hanlon et al. (2010) performed simultaneous CGMALS and CG-DLS measurements to enhance plate reader-based CG-DLS.

\section{Methods and expected signals}

Composition-gradient-MALS quantifies self-association via the concentration dependence of $\mathrm{M}_{\mathrm{w}}$, and hetero-association via the compositional dependence of $\mathrm{M}_{\mathrm{w}}$. When necessary, the two types of analyses may be combined in a single run, and the total data set is fit to Eqs. 3-5 constructed to represent all species and complexes present.

Figure 1 illustrates a three-part method comprising concentration gradients of each of two proteins individually for analyzing their respective molar masses and selfinteractions and a composition gradient for analyzing further hetero-association. Individual segments may be included or omitted as necessary, and other forms of composition gradients may be desirable under specific circumstances. Selfassociation analysis beyond that of the monomer-dimer requires a greater range of concentrations.

The functional forms expected for different selfassociation models corresponding to the gradients of Fig. 1a, c differ not only in the overall light scattering signal but also in the shape of the curvature, so that judicious curve fitting discriminates models that produce overall similar levels of scattered intensity, as illustrated in Fig. 2a. It should be noted that the $10 \mu \mathrm{M}$ monomer-trimer association differs from the dimer models both in overall height, since 


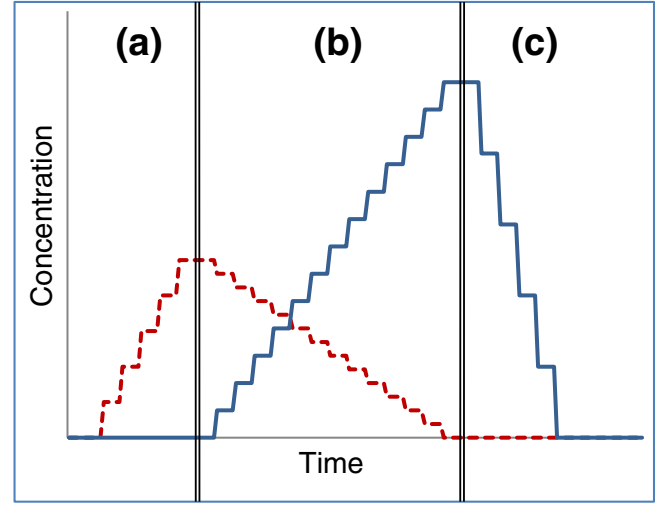

Fig. 1 Method for analyzing simultaneous self- and hetero-interactions. Solid blue line Protein X, broken red line Protein Y. a selfinteraction of $\mathrm{Y}$, b hetero-interaction $\mathrm{X}+\mathrm{Y}$, $\mathbf{c}$ self-interaction of $\mathrm{X}$. After Attri and Minton 2005b

the trimer molar mass exceeds that of the dimer, and in shape, due to different functional dependences on concentration.

When the molar masses of hetero-associating species do not differ by more than a factor of $\sim 5-10$, a 'crossover' composition gradient as in Fig. $1 \mathrm{~b}$ is optimal in that the contrast between interacting and non-interacting light scattering signals is prominent and intuitive. As depicted in Fig. 2b, complex formation leads to a peak in the MALS signal at the stoichiometric composition ratio; the height of the peak increases with affinity since a larger mole fraction of complexes produces higher solution molar mass. Figure $2 \mathrm{c}$ illustrates how the position and height of the peak, as well as the shape of the curve, correspond to the absolute complex stoichiometry.

When the mass ratio of the interacting species exceeds 5 10:1, a titration-like gradient-holding the concentration of the larger molecule constant and increasing concentrations of the smaller molecule-is preferable to the linear crossover, preventing small uncertainties in the concentration of the larger molecule from overwhelming the change in solution $\mathrm{M}_{\mathrm{w}}$ due to binding of a small molecule to a large one. Composition gradients may be optimized for signal contrast via simulations, such as using the Calypso software tools.

\section{Results}

The experiments reported below all pertain to automated CG-MALS, usually in the stopped-flow configuration. They are presented in order of increasing complexity of association models.

\section{Self-association}

Analysis of multiple self-association states can be quite challenging in that the same concentration dependence may be fit by more than one model, especially if the data
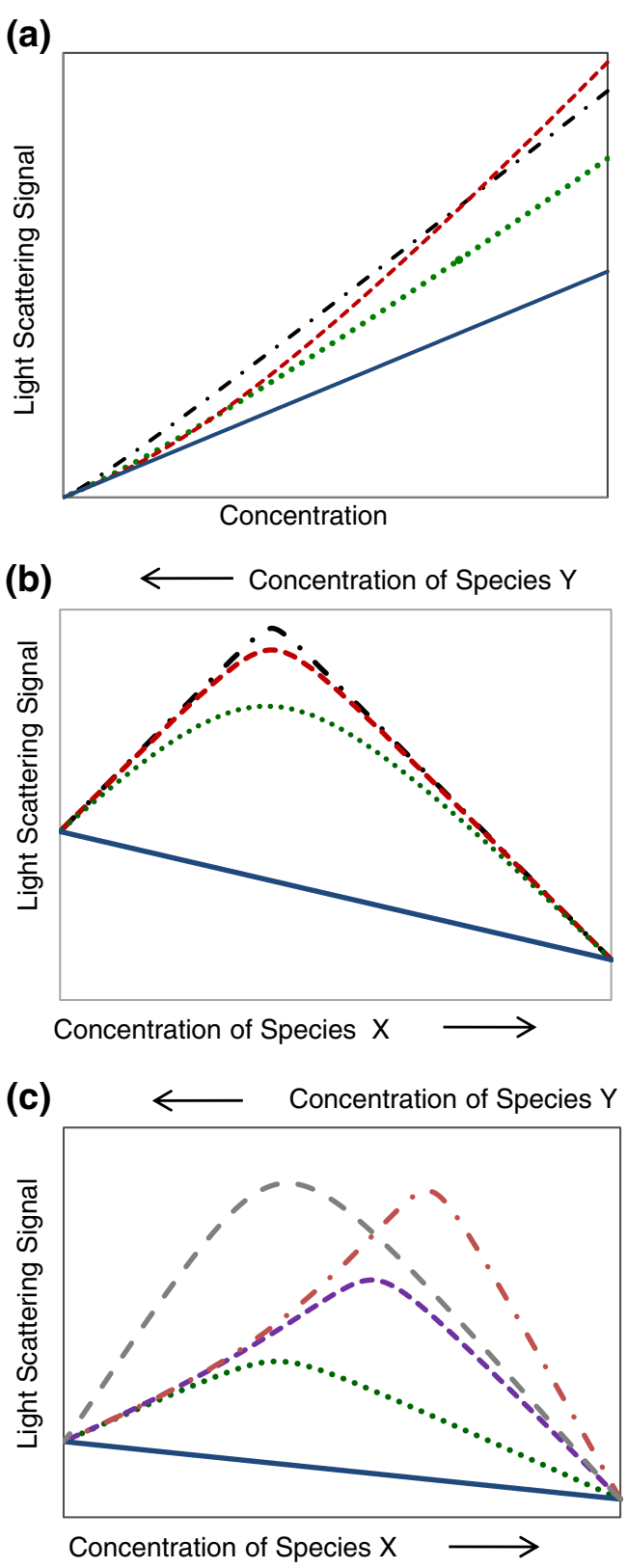

Fig. 2 Simulated light scattering (LS) signals for: a single-species concentration gradients (solid blue line no interaction, dotted green line monomer-dimer $\mathrm{K}_{\mathrm{D}}=10 \mu \mathrm{M}$, dashed red line monomer-trimer $\mathrm{K}_{\mathrm{D}}=10 \mu \mathrm{M}$, dash-dot black line monomer-dimer $\mathrm{K}_{\mathrm{D}}=1 \mu \mathrm{M}$ ), b crossover gradient of Fig. $1 \mathrm{~b}$ assuming 1:1 binding at three different affinities (solid blue line no interaction, dotted green line $\mathrm{K}_{\mathrm{D}}=100 \mathrm{nM}$, dashed red line $\mathrm{K}_{\mathrm{D}}=10 \mathrm{nM}$, dash-dot black line $\mathrm{K}_{\mathrm{D}}=1 \mathrm{nM}$ ), c crossover gradient of Fig. $1 \mathrm{~b}$ assuming different stoichiometries (protein $\mathrm{X}$ : protein $\mathrm{Y}$ ) at constant per-site binding affinity [solid blue line no interaction, dotted green line 1:1, dashed purple line 2:1, dash-dot red line 3:1, long-dash-gray line $2: 2\left(\mathrm{~K}_{\mathrm{D}}\right.$ for $2: 2$ is $10 \times$ that of the other stoichiometries)]

are acquired over a limited concentration range. At such times all that may be left is to apply Occam's razor, postulating that the simplest model that fits the empirical data is usually the correct one. Del Castillo et al. (2011) characterized the effect of nucleotides and the M-domain on self- 
association of $\mathrm{ClpB}$, a hexameric molecular chaperon, by means of CG-SLS, SE, and other techniques. CG-SLS and SE were in good agreement that the simplest, best-fit model to this self-association is monomer-hexamer-dodecamer, and that the addition of ATP and ADP enhanced oligomerization by $5 \times$ and $8 \times$, respectively.

FtsZ, a bacterial cell division protein that self-associates to produce protofilaments in the form of chains and rings, has been the subject of several CG-MALS studies. Kameyama and Minton (2006) and Martos et al. (2010) found that FtsZ oligomerization is best fit to a quasi-isodesmic association model, meaning that the protein molecules form dimers, trimers, tetramers, etc., with a nearly constant affinity to bind new monomers to the growing chain. Exchanging EDTA for $\mathrm{Mg}^{2+}$ in the guanosine 5'-diphosphate (GDP)-containing buffer increased the affinity by threefold. The best-fit model and equilibrium association constants were essentially identical between CG-MALS and SE.

Guanosine 5'-triphosphate (GTP) induces extensive polymerization of FtsZ. Monterroso et al. (2012a, b) employed CG-MALS, CG-DLS, sedimentation velocity, and cryotransmission electron microscopy (TEM) to investigate filaments formed by FtsZ in the presence of GTP and a slowly-hydrolyzing GTP analog, GMPCPP, at multiple concentrations of $\mathrm{Mg}^{2+}$. These authors found that the distributions of mass and size correlated to a cyclic polymer with a favored configuration that depended on the specific nucleotide as well as $\mathrm{Mg}^{2+}$ concentration; the affinities varied widely, from $\sim 60 \mu \mathrm{M}$ to $1 \mathrm{nM}$. At $5 \mathrm{mMMg}^{2+}$, FtsZ formed rings with a narrow distribution of sizes, found by CGMALS to correspond to $130 \pm 20$ monomers and an $r_{g}$ of $100 \pm 10 \mathrm{~nm}$ (GTP), or to $190 \pm 20$ monomers and an $r_{\mathrm{g}}$ of $110 \pm 11 \mathrm{~nm}$ (GMPCPP). These dimensions correlate well to the expected size of a ring constructed out of the corresponding number of monomers. DLS diffusion constants, sedimentation coefficients, and cryo-TEM studies all supported these findings, as did the atomic force microscopy images (Mateos-Gil et al. 2012).

As an important biotherapeutic, insulin self-association is of great practical interest. Attri et al. (2010a, b) characterized oligomerization of insulin in the presence of and in the absence of $\mathrm{Zn}$ by means of CG-MALS and CG-DLS. In the absence of $\mathrm{Zn}$, insulin appears to follow an isodesmic association model with a minimal $\mathrm{K}_{\mathrm{D}}$ of $40 \mu \mathrm{M}$ at pH7.2; SE data at pH7.0 yielded an essentially identical affinity. At pH10 the best-fit model differed slightly, indicating that the initial dimer nucleation step is about fivefold weaker than the addition of subsequent monomers. In the presence of $\mathrm{Zn}$ and neutral $\mathrm{pH}$, insulin is known to form hexamers with the hexamer association complete beyond $\sim 0.3 \mathrm{mg} / \mathrm{mL}$ (Milthorpe et al. 1977). The present study shows that insulin hexamers further selfassociate isodesmically with a $\mathrm{K}_{\mathrm{D}}$ of just under $100 \mu \mathrm{M}$.

Weak self-association that is only evident at concentrations above $\sim 1 \mathrm{~g} / \mathrm{L}$ requires extended treatment to account for non-specific repulsion, or 'thermodynamic non-ideality'. In addition, making such measurements in a stopped-flow CG-MALS apparatus may be challenging due to the high viscosity of concentrated solutions, plugging of in-line filters, or high sample consumption. Fernández and Minton (2009) measured the self-association of chymotrypsin (CTR) at concentrations up to $70 \mathrm{~g} / \mathrm{L}$ and various $\mathrm{pH}$ values by means of the in-place titration system described above. Minton (2007) argued for an analysis which assumes thatat sufficient ionic strength where long-range electrostatic repulsion is screened-non-specific/repulsive interactions can be described by an effective hard sphere model, and the effective specific volumes of all oligomers are equal to that of the monomer. While the homodimerization of CTR had been previously well-characterized up to several grams per liter, a new, weak self-association state was found to exist beyond $10 \mathrm{~g} / \mathrm{L}$ - that of the pentamer or hexamer with a $K_{D}$ on the order of several hundred micromoles/Liter.

Small molecule-protein binding is not usually directly accessible to CG-MALS since the change in the $M_{w}$ of the solution upon binding will be irresolvable. However, the effect of small molecules on protein-protein interactions often can be addressed. Some and Hanlon (2010) monitored the dissociation kinetics of $\alpha$-chymotrypsin dimers upon exposure to AEBSF, a small molecule inhibitor. The initial concentration of CTR contained about $25 \%$ dimers in
Fig. 3 Multi-angle static light scattering (MALS) signal showing slow, multi-domain assembly kinetics. FliM, FliG Multi-domain flagellar motor proteins. Dotted black line FliG $_{\mathrm{mc}}$ concentration, dashed red line $\mathrm{FliM}_{\mathrm{nm}}$ concentration, solid blue line LS signal. Inset Intermediate assembly state derived from quasi-equilibrium analysis of MALS data, 1 $\mathrm{h}$ after mixing

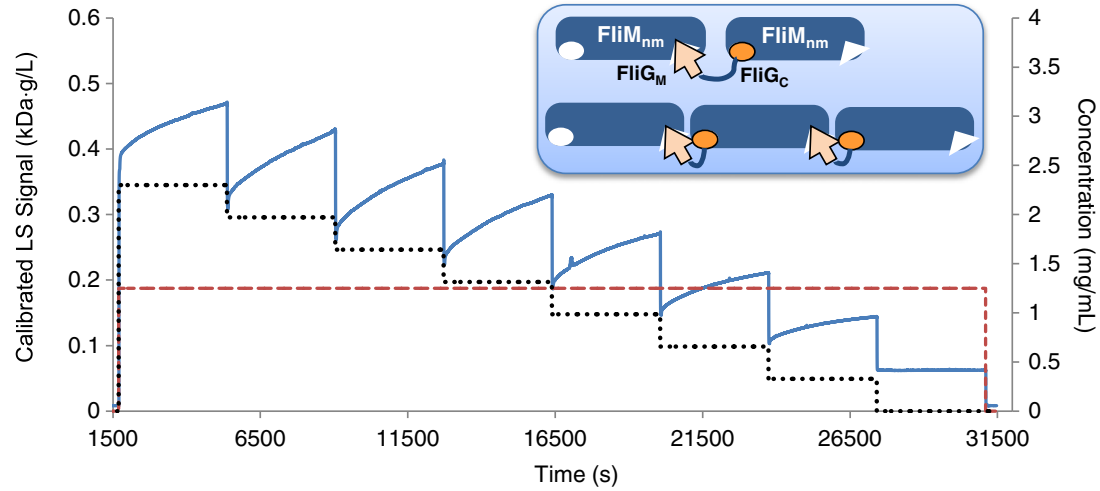


equilibrium with monomers. Upon introduction of AEBSF, the dimers slowly dissociated such that the $\mathrm{M}_{\mathrm{w}}$ approached that of a purely monomeric protein solution. The dependence of this dissociation rate on AEBSF concentration led to determination of the relevant kinetic parameters.

The smallest molecule so far reported to have been characterized for self-association by CG-MALS is insulin $\left(\mathrm{M}_{\mathrm{w}}=\right.$ $5700 \mathrm{Da})$. Work is ongoing to study reversible selfassociation of peptides with molar masses of 1-4 kDa.

\section{Hetero-association}

Pioneering work in automated CG-MALS of the heteroassociating proteins CTR and STI was reported by Attri and Minton (2005b). At pH 7.2 the self-association of CTR is weak, allowing these authors to ignore homodimers. However, a complete analysis of the equilibrium states must include 1:1 and 1:2 complexes and also inactive monomers of one or both species which, due to misfolding or other alterations, are incompetent to participate in the interaction. Fitting to different association models ruled out a simple 1:1 association, while a bivalent model assuming equivalent and independent (non-cooperative) binding sites did fit the data, especially when an incompetent fraction of CTR was allowed. The single-site affinity $\mathrm{K}_{\mathrm{D}}=330 \mathrm{nM}$ was in good agreement with SE data acquired under similar conditions, and the inactive fraction of previously lyophilized CTR was estimated at $30 \%$.

Simultaneous self- and hetero-association is amenable to CG-MALS analysis, as demonstrated by Kameyama and Minton (2006) for the interaction of CTR with bovine pancreatic trypsin inhibitor (BPTI) over a range of $\mathrm{pH}$ conditions that modulates CTR self-association. CTR and BPTI form a simple 1:1 complex, but 2:1 complexes due to BPTI binding to a CTR dimer were ostensibly not observed, indicating competition for the same site or at least complete mutual inhibition. Association constants of simultaneously occurring homodimers and heterodimers were calculated over the $\mathrm{pH}$ range 4.4-8.0.

Excipients that interfere with isothermal titration calorimetry (ITC) may not be detrimental to CG-MALS. Zhu et al. (2010) studied the interaction between a tau protein fragment and $7-\mathrm{kDa}$ heparin in the presence of DTT, a reducing agent that is incompatible with ITC. CG-MALS indicated a simple $1: 1$ association with $K_{D}=330 \mathrm{nM}$, complementing the results of the ITC analysis in the absence of DTT.

The bacterial cell division protein FtsZ not only selfassociates reversibly as filaments, but also binds in vitro to ZipA, a membrane protein believed to be a part of the cell division machinery. Martos et al. (2010) investigated this hetero-association by means of a solubilized fragment, sZipA. The results of both the CG-MALS and SE analyses support the interpretation that only one monomer of sZipA binds to any given FtsZ protofilament, giving credence to the purported role of ZipA as a membrane anchor. The binding affinity of sZipA to the FtsZ chains was found to increase by approximately fourfold, from $\mathrm{K}_{\mathrm{D}} \sim 6 \mu \mathrm{M}$ to $\sim 1.6 \mu \mathrm{M}$, when $1 \mathrm{mM}$ EDTA was replaced with $5 \mathrm{mM}$ $\mathrm{Mg}^{2+}$ in the buffer.

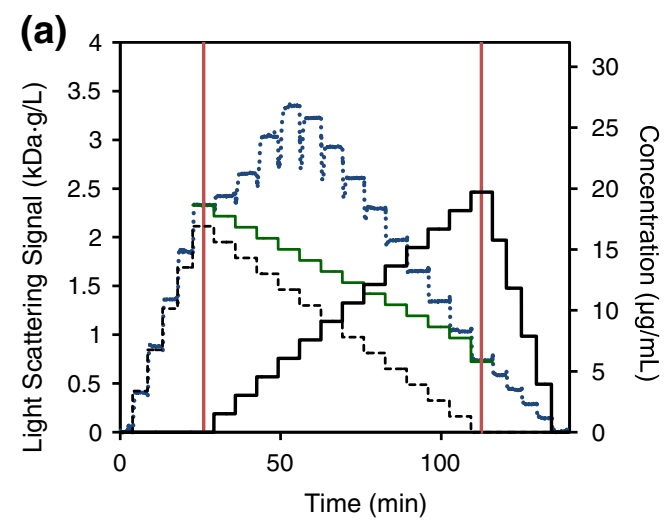

(b) Total Antibody Concentration (nM)

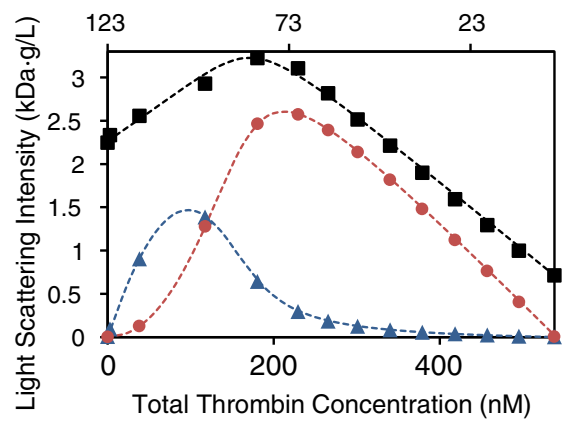

(c) Total Antibody Concentration (nM)

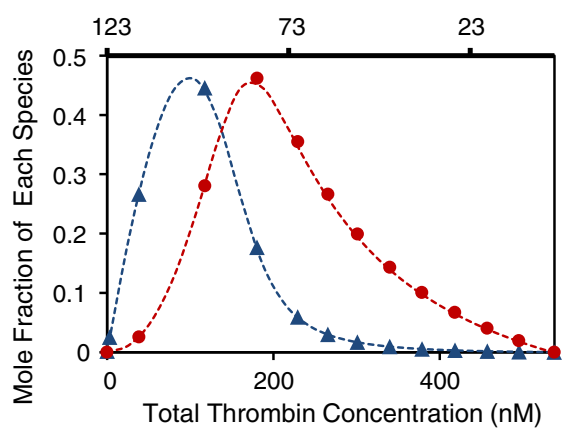

Fig. 4 Typical antibody-antigen binding data. a Composition-gradient (CG)-MALS data based on the method of Fig. 1. Dotted blue line Measured LS data, dashed black line injected anti-thrombin immunoglobulin G (ATI) concentration, solid black line injected $\alpha$-thrombin (THR) concentration, solid green line simulated no-interaction LS signal. b Hetero-association modeling. Black squares LS data points measured after equilibration, dashed black line best-fit model, 2 (THR):1(ATI) with equivalent and independent binding sites, $\mathrm{K}_{\mathrm{D}}=$ $9 \mathrm{nM}$. Other plots represent LS contributions from the complexes. Blue triangles 1:1, red circles 2 (THR):1 (ATI). c Mole fraction of complexes, same symbols as for $\mathbf{b}$. In $\mathbf{b}$ and $\mathbf{c}$, plots for free monomers are not shown 
Flagellar motor proteins self-assemble to form a highly organized motor structure. Kenrick et al. (2012a) investigated subunit assembly of the multi-domain flagellar motor proteins FliM and FliG by means of CG-MALS. In isolation, the middle domain of FliG binds to a site on the FliM middle domain with a $\mathrm{K}_{\mathrm{D}}=600 \mu \mathrm{M}$ (confirming nuclear magnetic resonance analysis by Vartanian et al. 2012), while the $\mathrm{C}$-terminal domain of FliG binds to a neighboring site on FliM with a $K_{D}=6 \mu \mathrm{M}$. The interactions of both FliG domains reach equilibrium within less than $20 \mathrm{~s}$, forming 1:1 complexes.

The association between the FliG protein expressed with both domains and FliM is qualitatively distinct from singledomain interactions, illustrating the evolution of a biochemical system from simple precursors to higher-order complexity. Figure 3 presents the CG-MALS data from a fixed concentration of FliM and varying concentrations of FliG. The stopped-flow delay time after each injection is $1 \mathrm{~h}$. Equilibration of the association process is dramatically slower than the individual FliM domain binding event. A quasi-equilibrium analysis of the 1-h data points provided a best-fit model of 1:2+2:3 (FliM:FliG) with average per-site affinity of $10 \mu \mathrm{M}$, as illustrated in Fig. 3 (inset); presumably, had the reactions been permitted to continue, the complexes would have consisted of longer FliM-FliG chains. While we do not expect the observed kinetics to be biologically relevant - the in vivo process of motor assembly could be assisted by chaperones or otherwise affected by the cellular environment - it is striking that the $\mathrm{C}$-ring assembly process initiates in vitro, even in the absence of supporting biological machinery and scaffolding.

For best efficacy, biotherapeutics are expected to bind to their targets with high affinity, generally leading to $\mathrm{K}_{\mathrm{D}}$ values in the low nanomolar range and down to the picomolar range. CG-MALS sensitivity is proportional to molar mass, permitting analysis of sub-nanomolar antibody-antigen or antibodyreceptor interactions when state-of-the-art detectors are combined with well-prepared solutions (smaller proteins, such as CTR, STI, FtsZ, and sZipA would only be quantifiable down

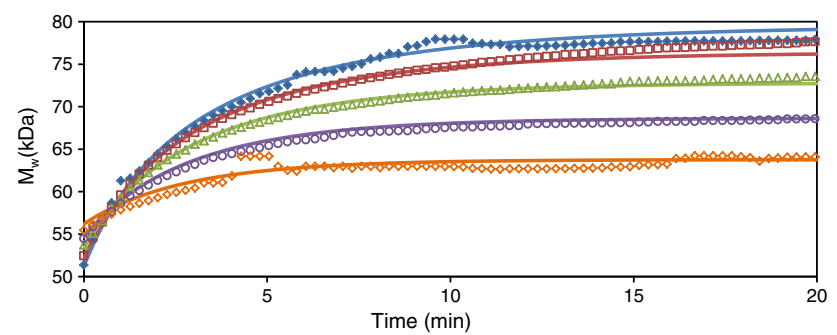

Fig. 5 THR-anti-thrombin (AT) binding kinetics, fixed AT concentration of $60 \mu \mathrm{g} / \mathrm{mL}$, and varying THR concentrations. Symbols represent measured values: solid blue diamonds $28 \mu \mathrm{g} / \mathrm{mL}$ THR, red squares $22 \mu \mathrm{g} / \mathrm{mL}$ THR, green triangles $17 \mu \mathrm{g} / \mathrm{mL}$ THR, purple circles $11 \mu \mathrm{g} /$ $\mathrm{mL}$ THR, open orange diamonds $5.6 \mu \mathrm{g} / \mathrm{mL}$ THR. Lines are best-fit to second-order kinetics; $1: 1$ binding with $\mathrm{K}_{\mathrm{on}}=6.09 \times 10^{-3} \mathrm{~s}^{-1} \mathrm{M}^{-1}$ to single-digit nanomolar $\mathrm{K}_{\mathrm{D}}$ ). We have characterized several such interactions by CG-MALS, the tightest so far at $\mathrm{K}_{\mathrm{D}}=$ $0.3 \mathrm{nM}$. Of the non-proprietary samples we have measured, $\alpha$ thrombin (THR) and anti-thrombin immunoglobulin G (ATI) have so far presented the highest affinity at $9 \mathrm{nM}$ per binding site (Fig. 4), comparing favorably to $15 \mathrm{nM}$ determined by enzyme-linked immunosorbent assay (ELISA) (data available at: Haematologic Technologies, Essex Junction, VT). In contrast to ELISA, surface plasmon resonance (SPR), and most other techniques suitable for this affinity range, CG-MALS not only shows unequivocally the 1:2 stoichiometry of an antibody-antigen interaction (SPR provides, at best stoichiometric ratio; see Rich and Myszka 2008 for the ambiguity inherent in SPR stoichiometry analyses) but also the presence of, or lack of other complexes such as homo-oligomers of either species or higher order hetero-complexes.

Kenrick et al. (2012b) extended the THR-ATI analysis to demonstrate how CG-MALS may characterize a three-body interaction. Anti-thrombin (AT; Haematologic Technologies)
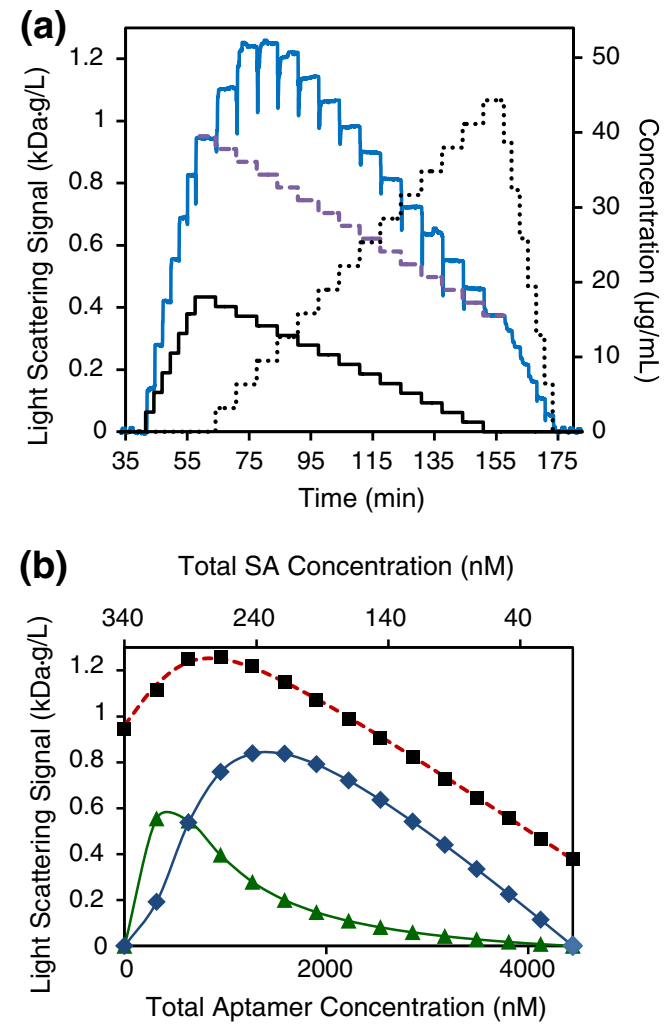

Fig. 6 a CG-MALS aptamer-streptavidin (APT-SA data). Solid blue line Measured LS, dashed purple line simulated no-interaction data, solid black line injected SA concentration, dotted black line injected APT concentration. b Hetero-association modeling. Black squares Measured LS, dashed red line best-fit model of 2 APT:1 SA, equivalent and independent binding sites, $\mathrm{K}_{\mathrm{D}}=190 \mathrm{nM}$, green triangles $1: 1$, blue diamonds 2:1 complex. LS from free monomers not shown. In $\mathbf{b}$ lines (except best-fit model) are guides to the eye, and the self-interaction data are excluded as no self-association was evident. Conversion of LS signals to units of kiloDaltons gram per liter is approximate because APT and SA have different specific refractive index increment $(\mathrm{dn} / \mathrm{dc})$ values 
binds irreversibly to THR, potentially inhibiting the interaction with ATI. The concentration-dependent kinetics of THRAT complexation were analyzed via CG-MALS (Fig. 5), exhibiting a 1:1 stoichiometric state with $\mathrm{k}_{\text {on }}=6.09 \times 10^{3} \mathrm{~s}^{-1}$ $\mathrm{M}^{-1}$, comparable to fluorescence-based measurements of $5.8 \times 10^{3} \mathrm{~s}^{-1} \mathrm{M}^{-1}$ (Izaguirre et al. 2007) and surface plasmon resonance measurements of $9 \times 10^{3} \mathrm{~s}^{-1} \mathrm{M}^{-1}$ (Elg and Deinum 2002). Approximately $23 \%$ of the AT was found to be inactive, similar to the manufacturer's data (21\%). For THR-AT-ATI interactions, a stock solution of THR-AT was prepared by incubating AT and an excess of THR for $1 \mathrm{~h}$ and then removing the inactive/unbound AT and THR by size exclusion chromatography. The THR-AT complex eluent was then combined with ATI to determine the affinity and stoichiometry of AT-bound THR with ATI. While the best-fit model was unchanged at 2(THR): 1 (ATI), the KD increased from 9 to $300 \mathrm{nM}$, indicating a negative cooperativity most likely due to either steric hindrance or an allosteric reduction in the active site as per Gandhi et al. (2008).

CG-MALS works just as well for oligonucleotides and peptides as for proteins. Bing et al. (2010) identified a 10kDa streptavidin-binding DNA aptamer (APT). Since streptavidin (SA) exists as a native tetramer, the APT-SA stoichiometry potentially could be $1: 1,2: 1,3: 1$, or $4: 1-$ or larger if APT itself is multivalent or either species selfassociates. Kenrick et al. (2012c) measured APT-SA interactions in a full CG-MALS gradient, shown in Fig. 6. No evidence for self-association was found; the heteroassociation data proved the availability of exactly two equivalent and independent binding sites on SA for APT, $\mathrm{K}_{\mathrm{D}}=190 \mathrm{nM}$. The latter result suggests that SA acts as a dimer of dimers where APT may bind to the cleft site, as confirmed by mass spectroscopy (Ruigrok et al. 2012).
The hetero-association of two multivalent macromolecules holds the potential for assembly of relatively large complexes. Figure 7 illustrates some examples of structures that could form from two bivalent molecules (Fig. 7a) and from one bivalent and one dimerizing molecule (Fig. 7b). CG-MALS is well-suited to analyzing these interactions thanks to its determination of solution molar mass which helps distinguish complexes with the same stoichiometric ratio but different absolute stoichiometries. The extended association states can be modeled as 'metacomplexes', i.e., the product of selfassociation of hetero-associated base units. For example, the $n: n$ series of Fig. 7 a may be considered $(\mathrm{XY})_{1},(\mathrm{XY})_{2},(\mathrm{XY})_{3}$, etc. Often this type of metacomplexation may be modeled conveniently as an isodesmic self-association of the base units, either finite (terminating at some $n$-mer) if there is some limiting mechanism such as cyclization (ring formation), or infinite if the metacomplexation is open-ended. The association model is described by two association constants: $i X+j Y \stackrel{K_{D, 1}}{\rightleftarrows} X_{i} Y_{j}$ and $X_{i} Y_{j}+X_{i} Y_{j}+X_{i} Y_{j}+\ldots \stackrel{K_{D, 2}^{n-1}}{\rightleftarrows}\left(X_{i} Y_{j}\right)_{n}$, where $\mathrm{i}=\mathrm{j}=1$ for Fig. $7 \mathrm{a}$, and $\mathrm{i}=1, \mathrm{j}=2$ for Fig. $7 \mathrm{~b}$. A complete model should account for the additional complexes shown in Fig. 7.

Further evidence for the 'dimer of dimers' structure of SA, apparent in the crystallographic structure (Hendrickson et al. 1989), was found in its interaction with anti-streptavadin immunoglobulin G1 (ASI; kindly provided by S. Cao and J. Pollastrini, Amgen). CG-MALS data (Kenrick and Some 2012) indicated no self-association and peak SLS signals at a 1:1 stoichiometric ratio. However, as shown in Fig. 8a, the total light scattering intensity far exceeded that expected for the 1:1,2:1, or 1:2 states. In a first-pass analysis the data are fit well by a model of infinite self-association of 1:1 sub-units, with an intra-sub-unit $\mathrm{K}_{\mathrm{D}, 1}$ of $22 \mathrm{nM}$ and inter-sub-unit $\mathrm{K}_{\mathrm{D}, 2}$ of $50 \mathrm{nM}$. One might expect $\mathrm{K}_{\mathrm{D}, 1}=\mathrm{K}_{\mathrm{D}, 2}$, since the sub-units

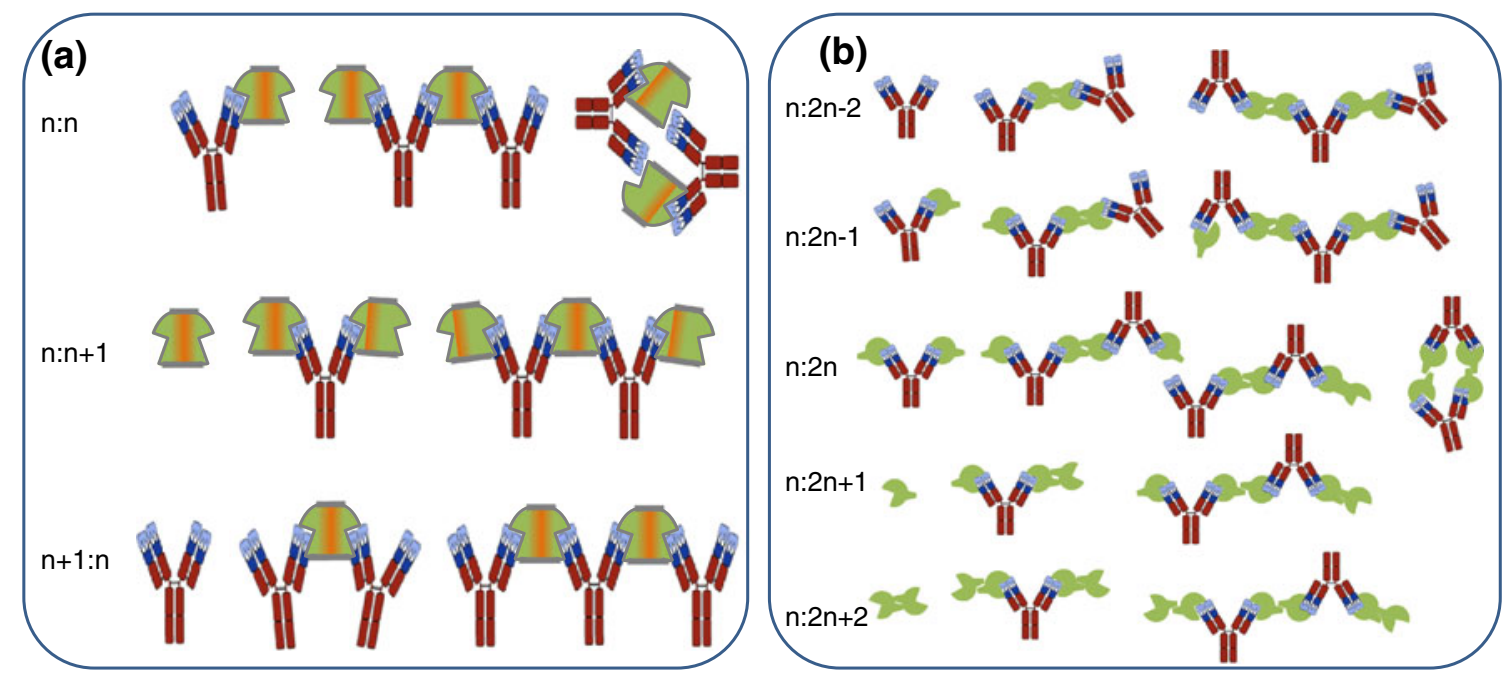

Fig. 7 Molecular networks that may form from multivalent molecules. a Bivalent antibody (Ab) and bivalent antigen (Ag). b Bivalent antibody and monovalent, self-associating antigen. Stochiometries represent Ab:Ag 
bind at the same sites that participate in intra-unit association. The lower inter-unit affinity could represent cooperativity that disfavors larger metacomplexes; back-calculation of the concentrations of each metacomplex shows that only $(1 \mathrm{SA}: 1 \mathrm{ASI})_{1},(1 \mathrm{SA}: 1 \mathrm{ASI})_{2}$, and $(1 \mathrm{SA}: 1 \mathrm{ASI})_{3}$ appear at any appreciable level under these conditions. Further investigation of this system should include composition gradients at higher overall concentrations to test for correspondingly higher association states.

A more complete analysis, presented in Fig. 8b, c, includes the additional $n+1: n$ and $n: n+1$ complexes that should exist in these networks alongside $n: n$ complexes. Inclusion of those terms not only produces a somewhat better fit-with 1:2, 2:1, and 2:3 terms contributing significantly to the total light scattering - but the $\mathrm{K}_{\mathrm{D}}$ determined from the associations constants of all complexes converge to the narrow range of $20 \pm 5 \mathrm{nM}$, suggesting no cooperativity.

CG-MALS analysis cannot distinguish conclusively between possible mechanisms behind the apparent bivalent nature of tetrameric SA, such as steric inhibition or specificity of the ASI complementarity-determining region for epitopes located along SA's axis of symmetry. On the other hand, CG-MALS can be a quite powerful tool in confirming and fleshing out predictions based on structural models of the individual proteins, such as the expected 'dimer of dimers' behavior of SA (the author is not aware of attempts to predict this specific interaction).

In our experience metacomplex formation is often accompanied by relatively slow kinetics. The SA-ASI interaction does exhibit such slow kinetics, clearly evident in Fig. 8a. At this time we have not attempted to analyze the kinetics in this rather complicated system; however, the equilibrium result of essentially a single $\mathrm{K}_{\mathrm{D}}$ would suggest that one association rate and one dissociation rate could suffice to describe the entire temporal evolution.

CG-MALS is particularly powerful in addressing some of the quite complex interactions exhibited by biological systems. We investigated the Cre-loxP recombination system (purified enzyme and oligonucleotide kindly provided by K. Gupta and G.D. Van Duyne, University of Pennsylvania). Cre recombinase acts on loxP, a 35-bp sequence from bacteriophage $\mathrm{P} 1$, to effect site-specific insertions, deletions, translocations, and inversions of DNA in prokaryotic and eukaryotic cells. Previous work on this system utilizing electrophoretic mobility shifts assays (EMSA; Ringrose et al. 1998) and SPR (Rüfer et al. 2002) found that two Cre molecules bind to $\operatorname{lox} P$ with positive cooperativity. Although neither the protein nor DNA self-associate on their own, two CreloxP units further associate to form a 4:2 synaptic complex that is necessary for catalysis; this assembly was previously examined by SE and EMSA (Ghosh et al. 2007).

In a single three-hour CG-MALS run, we reached qualitatively similar conclusions as those obtained previously through these laborious and time-consuming processes, although a direct quantitative comparison has not yet been

(a)

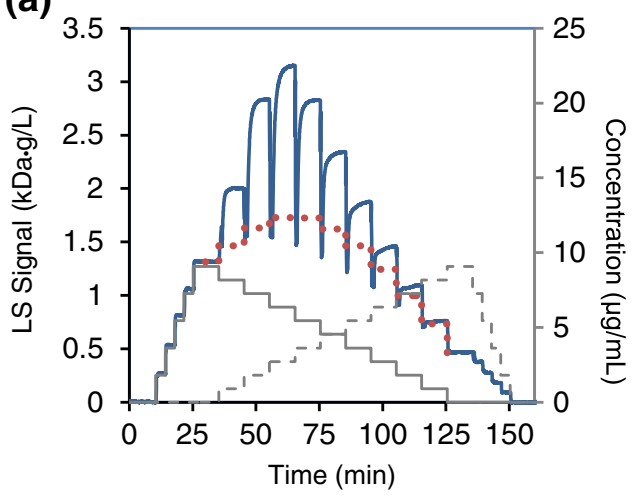

(b)

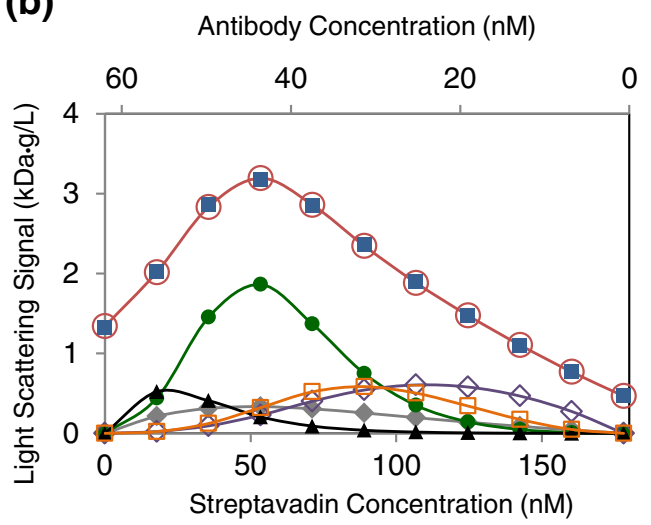

(c)

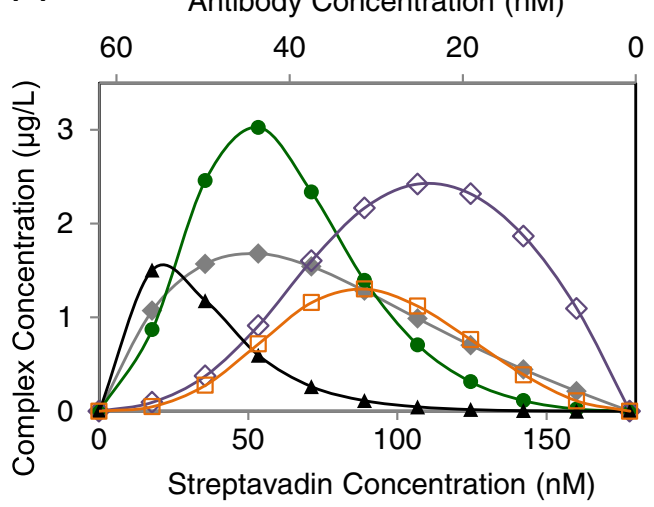

Fig. 8 a CG-MALS gradient of multi-valent/multi-valent SA+ immunoglobulin G interaction. Solid blue line MALS data, dotted red line simulated LS from 1:2 interaction, solid gray line injected SA concentration, dashed gray line injected antibody concentration. b Heteroassociation fitting. Blue squares LS data from each injection measured at equilibrium, open red circles best fit to a model assuming the complexes of Fig. $7 \mathrm{a}$, single-site $\mathrm{K}_{\mathrm{D}}=20 \mathrm{nM}$, filled gray diamonds $1: 1$, filled green circles $2: 2+3: 3+4: 4+\ldots$, closed black triangles 1 SA:2 antibody, open purple diamonds 2 SA:1 antibody, open orange squares $3 \mathrm{SA}: 2$ antibody. c Solution concentrations of the individual complexes, symbols as in $\mathbf{b}$. Filled green circles represent total mass concentration of all $n: n$ complexes excluding 1:1. In $\mathbf{b}$ and $\mathbf{c}$ free monomer plots not shown, lines are guides to the eye, and the selfinteraction data are excluded as no self-association was evident 
possible because of variations in the specific oligonucleotide sequence and length, catalytic mutations, and buffers. CGMALS analysis indicated that, at $\mathrm{pH} 7.5$, the first Cre attaches to $\operatorname{lox} P$ with a $\mathrm{K}_{\mathrm{D}, 1}=30-170 \mathrm{nM}$ and the second Cre binds at an approximately tenfold greater affinity (4-17 nM), while the final reaction $\mathrm{Cre}_{2} \operatorname{loxP}+\mathrm{Cre}_{2} \operatorname{loxP} \Leftrightarrow$ $\mathrm{Cre}_{4} \operatorname{loxP}_{2}$ occurs with $\mathrm{K}_{\mathrm{D} \text {,synap }}=380 \mathrm{nM}$. For comparison, SPR determined $\mathrm{K}_{\mathrm{D}, 1}$ on the order of $6.5 \mathrm{nM}$ and $\mathrm{K}_{\mathrm{D}, 2}$ of mid- to low-pM for $\sim 100-2000 \times$ cooperativity, while EMSA found a cooperativity factor of only 14; SE and EMSA determined $K_{D \text {,synaps }}$ in the range of $10-14 \mathrm{nM}$. Association kinetics and the $r_{g}$ sizes of the complexes, although available in the LS data, were not analyzed.

\section{Summary}

The real power of light scattering for the analysis of biomolecular interactions lies in a very basic yet essential aspect: the determination of solution molar mass and size, providing a direct window on simple or complex association states with minimal guesswork as to the meaning and interpretation of the observed signals. Future developments can be expected to include extending the range of equilibrium models; integrating the analysis of equilibrium and kinetics in order to further quantify site-specific interactions; integrating CG-MALS with CG-DLS analysis to facilitate lowvolume, rapid determination of thermodynamic parameters via van t'Hoff analysis in the DLS plate reader

The unique capabilities of CG-MALS and CG-DLS suggest that these techniques are destined to become essential tools in the hands of the physical biochemist. Researchers already utilizing batch light scattering for fundamental physical biochemistry will find that the automated apparatus increases their productivity and range of applications by an order of magnitude, while those utilizing other biophysical techniques, such as SPR or ITC, will discover the complementarity between these methods. Given its particular suitability to quantifying multispecies complexes, oligomerization, and aggregation under a large range of solvent conditions, one can envision CG-MALS pervading all fields of biological interactions from essential genetic manipulation and cellular machinery through the biology of disorders, such as beta amyloid aggregation or immune responses to viral agents.

Acknowledgments In addition to those cited above, Sophia Kenrick also carried out the Cre-loxP CG-MALS analysis; her input to this manuscript was invaluable. Another critical review was provided by Chris Broomell. Kushol Gupta assisted in describing the Cre-loxP system and results.

Most of this exciting work would not have been possible without the pioneering efforts of Allen P. Minton in the conception, implementation, and validation of automated CG-MALS. Allen's vision, innovation, and perseverance in developing novel, theoretically rigorous biophysical techniques for characterizing protein-protein interactions have been an inspiration to my colleagues at Wyatt Technology and myself. Our collaboration arose because Allen has never been content to merely publish: he is driven to provide better tools for the entire biophysical community. It has been my privilege to enjoy not only his tutelage and mentorship, but also the warm friendship of both Allen and his wife Sima. Selection for this special edition of Biophysical Reviews was a deeply appreciated honor. I look forward to additional productive interactions with Allen in developing new products for the benefit of the scientific community.

Conflict of interest The author is an employee of Wyatt Technology Corp. and is actively involved in the development and promotion of the Calypso CG-MALS instrumentation and software.

Ethical standards All experiments performed by or in association with the author comply with relevant laws of the USA.

Open Access This article is distributed under the terms of the Creative Commons Attribution License which permits any use, distribution, and reproduction in any medium, provided the original author(s) and the source are credited.

\section{References}

Attri AK, Minton AP (2005a) New methods for measuring macromolecular interactions in solution via static light scattering: basic methodology and application to nonassociating and selfassociating proteins. Anal Biochem 337:103-110. doi:10.1016/ j.ab.2004.09.045

Attri AK, Minton AP (2005b) Composition gradient static light scattering (CG-SLS): a new technique for rapid detection and quantitative characterization of reversible macromolecular hetero-associations in solution. Anal Biochem 337:103-110. doi:10.1016/j.ab.2005.08.013

Attri AK, Fernández C, Minton AP (2010a) pH-dependent selfassociation of zinc-free insulin characterized by concentrationgradient static light scattering. Biophys Chem 148:28-33. doi:10.1016/j.bpc.2010.02.002

Attri AK, Fernández C, Minton AP (2010b) Self-association of Zninsulin at neutral $\mathrm{pH}$ : investigation by concentration-gradient static and dynamic light scattering. Biophys Chem 148:23-27. doi:10.1016/j.bpc.2010.02.001

Bajaj H, Sharma VK, Kalonia D (2007) A high-throughput method for detection of protein self-association and second virial coefficient using size-exclusion chromatography through simultaneous measurement of concentration and scattered light intensity. Pharm Res 24:2071-2083. doi:10.1007/s11095-007-9345-x

Bing T, Yang X, Mei H, Cao Z, Shangguan D (2010) Conservative secondary structure motif of streptavidin-binding aptamers generated by different laboratories. Bioorg Med Chem 18:1798-1805. doi:10.1016/j.bmc.2010.01.054

Burchard W, Cowie JMG (1972) Selected topics in biopolymeric systems. In: Huglin MB (ed) Light scattering from polymer solutions. Academic, London \& New York, pp 725-787

Das S, Stivison E, Folta-Stogniew E, Oliver D (2008) Reexamination of the role of the amino terminus of SecA in promoting its dimerization and functional state. J Bacteriol 190:7302-7307. doi:10.1128/JB.00593-08

del Castillo U, Alfonso C, Acebron SP, Martos A, Moro F, Rivas G, Muga A (2011) A quantitative analysis of the effect of nucleotides and the $\mathrm{M}$ domain on the association equilibrium of $\mathrm{ClpB}$. Biochem 50:1991-2003. doi:10.1021/bi101670s 
Elg S, Deinum J (2002) The interaction between captured human thrombin and antithrombin studied by surface plasmon resonance, and the effect of melagatran. Spectr Biomed Appl 16:257-270. doi: $10.1155 / 2002 / 673974$

Fernández C, Minton AP (2008) Automated measurement of the static light scattering of macromolecular solutions over a broad range of concentrations. Anal Biochem 381:254-257. doi:10.1016/ j.ab.2008.06.032

Fernández C, Minton AP (2009) Static light scattering from concentrated protein solutions ii: experimental test of theory for protein mixtures and weakly self-associating proteins. Biophys $\mathrm{J}$ 96:1992-1998. doi:10.1016/j.bpj.2008.11.054

Gandhi PS, Chen Z, Mathews FS, Di Cera E (2008) Structural identification of the pathway of long-range communication in an allosteric enzyme. Proc Natl Acad Sci USA 105:1832-1837. doi:10.1073/pnas.0710894105

Ghosh K, Guo F, van Duyne GD (2007) Synapsis of loxP sites by Cre recombinase. J Biol Chem 282:24004-24016. doi:10.1074/ jbc.M703283200

Hanlon AD, Larkin MI, Reddick RM (2010) Free-solution, labelfree protein-protein interactions characterized by dynamic light scattering. Biophys J 98:297-304. doi:10.1016/ j.bpj.2009.09.061

Hendrickson WA, Pähler A, Smith JL, Satow Y, Merritt EA, Phizackerley RP (1989) Crystal structure of core streptavidin determined from multiwavelength anomalous diffraction of synchrotron radiation. Proc Nat Acad Sci USA 86:2190-2194. doi:10.1073/pnas.86.7.2190

Izaguirre G, Swanson R, Raja SM, Rezaie AR, Olson ST (2007) Mechanism by which exosites promote the inhibition of blood coagulation proteases by heparin-activated antithrombin. J Biol Chem. 282:33609-33622. doi:10.1074/jbc.M702462200

Kameyama K, Minton AP (2006) Rapid quantitative characterization of protein interactions by composition gradient static light scattering. Biophys J 90:2164-2169. doi:10.1529/biophysj.105.074310

Kapoor N, Gupta R, Menon ST, Folta-Stogniew E, Raleigh DP, Sakmar TP (2010) Nucleobindin 1 is a calcium-regulated guanine nucleotide dissociation inhibitor of $\mathrm{G} \alpha_{\mathrm{i} 1}$. J Biol Chem 285:31647-31660. doi:10.1074/jbc.M110.148429

Kenrick S, Some D (2012) Self-association of antibody-antigen complexes quantified by CG-MALS and Wyatt Calypso ${ }^{\circledR}$ II. Faculty of 1000 F1000 Posters. Available at: http://f1000.com/posters/ browse/summary/1092810. Accessed 26 Dec 2012

Kenrick S, Vartanian A, Dahlquist F, Some D (2012a) Revealing the affinity of individual and combined FliG domains for FliM in the bacterial flagellar motor switch complex by CG-MALS. Faculty of 1000 F1000 Posters. Available at: http://f1000.com/posters/ browse/summary/1092808. Accessed 26 Dec 2012

Kenrick S, Lim V, Some D (2012b) Characterizing interactions between thrombin, antithrombin III, and an a-thrombin antibody by CGMALS. Faculty of 1000 F1000 Posters. Available at: http:// f1000.com/posters/browse/summary/1092828. Accessed 26 Dec 2012

Kenrick S, Ahmad K, Soh H, Some D (2012c) Quantifying affinity and stoichiometry of multivalent interactions by CG-MALS. Faculty of 1000 F1000 Posters. Available at: http://f1000.com/posters/browse/ summary/1092812. Accessed 26 Dec 2012. See also: Binding affinity and stoichiometry of multivalent macromolecular interactions by CG-MALS. Wyatt Technology Corp. Available at: http:// www.wyatt.com/files/literature/binding_affinity_stoichiometry_ calypso.pdf. Accessed 26 Dec 2012

Martos A, Alfonso D, Lopez-Navajas P, Ahijado-Guzman R, Mingorance J, Minton AP, Rivas G (2010) Characterization of self-association and heteroassociation of bacterial cell division proteins FtsZ and ZipA in solution by composition gradientstatic light scattering. Biochem 49:10780-10787. doi:10.1021/ bi101495x
Mateos-Gil P, Paez A, Horger I, Rivas G, Vicente M, Tarazona P, Velez M (2012) Depolymerization dynamics of individual filaments of bacterial cytoskeletal protein FtsZ. Proc Natl Acad Sci USA 109:8133-8138. doi:10.1073/pnas.1204844109

Milthorpe BK, Nichol LW, Jeffrey PD (1977) The polymerization pattern of zinc(II)-insulin at pH 7.0. Biochim Biophys Acta 495:195-202

Minton AP (2007) Static light scattering from concentrated protein solutions, I: general theory for protein mixtures and application to selfassociating proteins. Biophys J 93:1321-1328. doi:10.1529/ biophysj.107.103895

Monterroso B, Ahijado-Guzmán R, Reija B, Alfonso C, Zorrilla S, Minton AP, Rivas G (2012a) Mg2+-linked self-assembly of FtsZ in the presence of GTP or a GTP analogue involves the concerted formation of a narrow size distribution of oligomeric species. Biochem 51:4541-4550. doi:10.1021/bi300401b

Monterroso B, Rivas G, Minton AP (2012b) An equilibrium model for the $\mathrm{Mg}(2+)$-linked self-assembly of FtsZ in the presence of GTP or a GTP analogue. Biochem 51:4541-4550. doi:10.1021/bi300891q

Rich RL, Myszka DG (2008) Survey of the year 2007 commercial optical biosensor literature. J Mol Recognit 21:355-400. doi:10.1002/jmr.928

Ringrose L, Lounnas V, Ehrlich L, Buchholz F, Wade R, Stewart AF (1998) Comparative kinetic analysis of FLP and cre recombinases: mathematical models for DNA binding and recombination. J Mol Biol. 284:363-384. doi:10.1006/jmbi.1998.2149

Rüfer A, Neuenschwander PF, Sauer B (2002) Analysis of Cre-loxP interaction by surface plasmon resonance: influence of spermidine on cooperativity. Anal. Biochem. 308:90-99. doi:10.1016/S00032697(02)00247-6

Ruigrok VJ, Levisson M, Hekelaar J, Smidt H, Dijkstra BW, van der Oost J (2012) Characterization of aptamer-protein complexes by X-ray crystallography and alternative approaches. Int J Mol Sci 13:10537-10552. doi:10.3390/ijms130810537

Scherer TM, Liu J, Shire SJ, Minton AP (2010) Intermolecular interactions of IgG1 monoclonal antibodies at high concentrations characterized by light scattering. J Phys Chem B 114:1294812957. doi:10.1021/jp1028646

Sharma P, Rajalingam D, Kumar TKS, Singh S (2008) A light scattering study of the interaction of fibroblast growth factor (FGF) with its receptor. Biophys J 94:L71-L73. doi:10.1529/biophysj.108.129569

Some D, Hanlon A (2010) Characterizing protein-protein interactions via static light scattering: inhibition kinetics and dissociation. Am Biotech Lab 28:9-12

Some D, Kenrick S (2012) Characterization of protein-protein interactions via static and dynamic light scattering. In: Cai J, Wang RE (eds) Protein interactions. InTech, New York, pp 401-426. doi:10.5772/37240. http://www.intechopen.com/books/proteininteractions/characterization-of-protein-protein-interactions-viastatic-and-dynamic-light-scattering

Vartanian AS, Paz A, Fortgang EA, Abramson J, Dahlquist FW (2012) Structure of flagellar motor proteins in complex allows for insights into motor structure and switching. J Biol Chem 287:35779-35783. doi:10.1074/jbc.C112.378380

Woll MP, De Cotiis DA, Bewley MC, Tacelosky DM, Levenson R, Flanagan JM (2011) Interaction between the D2 dopamine receptor and neuronal calcium sensor- 1 analyzed by fluorescence anisotropy. Biochemistry 50:8780-8791. doi:10.1021/bi200637e

Wyatt PJ (1993) Light scattering and the absolute characterization of macromolecules. Anal Chim Acta 272:1-40. doi:10.1016/00032670(93)80373-S

Yamaguchi T, Adachi K (2002) Hemoglobin equilibrium analysis by the multiangle laser light-scattering method. Biochem Biophys Res Comm 290:1382-1387. doi:10.1006/bbrc.2002.6362

Zhu HL, Fernández C, Fan JB, Shewmaker F, Chen J, Minton AP, Liang Y (2010) Quantitative characterization of heparin binding to tau protein: implications for inducer-mediated tau filament formation. J Biol Chem 285:3592-3599. doi:10.1074/jbc.M109.035691 